Original Research Paper

\title{
A New Discussion of Different Noises and Filters
}

\author{
${ }^{1}$ Sundararajan, P.N. and ${ }^{2}$ S. Sasi Kumar \\ ${ }^{I}$ Department of Electronics and Communication Engineering, PSNA College of Engineering and Tech, Dindigul, \\ Tamilnadu, India \\ ${ }^{2}$ Department of Computer Science and Engineering, RMD Engineering College, Chennai, India
}

\author{
Article history \\ Received: 25-05-2014 \\ Revised: 25-06-2014 \\ Accepted: 30-12-2014 \\ Corresponding Author: \\ Sundararajan, P.N., \\ Department of Electronics and \\ Communication Engineering, \\ PSNA College of Engineering \\ and Tech, Dindigul, Tamilnadu, \\ India \\ E-mail: pnsundar74@yahoo.com
}

\section{Introduction}

Digital Image Processing is an element of digital signal processing. The field of digital image processing denotes to dealing with digital images by using a digital computer. Digital image processing is having various advantages over analog image processing; it permits a substantially broader ingathering of algorithms to be employed to input information and may maintain off of troubles for illustrating the development of noise and signal deformation throughout processing. Digital Image Processing demands the alteration of digital information to enhance the image qualities with the help of computer. The processing assists in enhancing the clarity, sharpness of image and details of characteristics of desire in the direction of extraction of data and foster investigation (Jain, 1989). Digital image processing is a wide subject and it frequently includes the protocols that may be composite mathematically, but the fundamental estimate behind digital image processing is less of complication. The digital image is afforded as input into a computer and computer is programmed to alter those information with the aid of an equation or with set of equations and then save the values of the computation for every pixel or picture component.

The results create a novel digital image which could be display or it may be taped in pictorial format or it can itself be foster altered by extra computer programs. To improve some characteristics in the information and to get rid of noise from image, the digital information is submitted to various image processing procedures (Gonzalez and Woods, 2005).
Image processing includes altering the quality of an image in order to:

- Enhance the pictorial data of an image for human interpretation

- Depict the image must be much desirable for freelance machine sensing

The methods of Image-processing can be sorted into principal three operational classes:

\section{Image Restoration}

It pays off for noise, data mistakes and the geometric aberration which is initialized while recording, scanning and the playback operations:

- It rejuvenates the occasional line gives up

- Utilized for repairing occasional line marking

- Beneficial for percolating of haphazard noise

- Improve geometric distortions

\section{Image Enhancement}

Image Enhancement Processing an image oriented figure out so that the consequence is much desirable for a specific application. Like sharpening or de-blurring an out of focus image, high spotting the boundaries of image, enhancing the contrast of image or raise the brightness degree of an image, getting rid of the noise from noisy image:

- Utilized for Contrast Improvement

- Intensity, saturation and hue transformations 
- Edge enhancement

- Developing the synthetic stereo image

\section{Various Sorces of Noise in Images}

Noise is brought in the image at the time of image attainment or transmission. Various components can be creditworthy for bringing up of noise in the image. The number of pixels sullied in the image will resolve the measurement of the noise. The primary origins of noise in the digital image are:

- The imaging sensor could be dissembled by environmental considerations throughout image attainment

- Deficient Light levels and sensor temperature might bring in the noise in the image

- Hindrance in the transmission channel would also spoil the image

- If dust particles are present on the scanner screen, they may also bring in noise in the image

\section{Image Noise}

Image noise is the haphazard fluctuation of brightness or color data in images developed by the sensor and circuitry of a scanner or digital camera. Image noise may also develop in film grain and in the inescapable shot noise of an ideal photon sensor (Boncelet, 2005). Image noise is usually considered as an unfavorable by-product of image capture. Even though those undesirable variations became recognized as "noise" by analogy with undesirable sound they are unhearable and such as dithering (Fig. 1). The types of Noise are following:

- Amplifier noise (Gaussian noise)

- Salt-and-pepper noise

- Shot noise (Poisson noise)

- Speckle noise

\section{Gaussian Noise (Amplifier Noise)}

The standard example of amplifier noise is additive, Gaussian, freelance at every pixel and free of the signal intensity. In color cameras wherein much amplification is utilized in the blue color channel rather than in the green or red channel, there may be much noise in the blue channel. Amplifier noise is a main component of the "read noise" of an image sensor, which is, of the constant noise level in dark areas of the image (Boncelet, 2005) Equation 1:

$$
p(z)=\frac{1}{\sqrt{2 \Pi \sigma}} e^{-(z-\mu)^{2 / 2 \sigma^{2}}}
$$

\section{Salt-and-Pepper Noise}

An image consists of salt-and-pepper noise may have dark pixels in bright areas and bright pixels in dark areas (Srinivasan and Ebenezer, 2007). This type of noise may be induced by dead pixels, analog-todigital converter faults, bit mistakes in transmission, etc. This may be got rid of in large part by utilizing dark frame subtraction and by estimating the value around dark/bright pixels:

$$
s(z)=\left\{\begin{array}{l}
S_{p} \text { for } z=p \\
S_{q} \text { for } z=q \\
0 \text { otherwise }
\end{array}\right.
$$

\section{Poisson Noise}

Poisson noise or shot noise is a kind of electronic noise which happens while the finite number of elements which contain energy, like electrons in an electronic circuit or photons in an optical device, is little plenty to develop to sensible statistical variations in quantification (Boncelet, 2005).

The PDF of Gaussian random variable is given by Equation 2:

$$
P(x)=1 /(\sigma \sqrt{2 \Pi})^{*} e^{(x-\mu)^{2}} / 2 \sigma^{2}-\infty<0<\infty
$$

Where:

$\mathrm{P}(\mathrm{x}) \quad=$ The Gaussian distribution noise in image $\mu$ and $\sigma=$ The mean and standard deviation respectively.

\section{Speckle noise}

Speckle noise is a type of granular noise which subsists in inherent manner and disrupts the quality of the dynamic radar and Synthetic Aperture Radar (SAR) images. Speckle noise in traditional radar is a consequence of haphazard variations in the return signal from an object which is not much huge than a single image-processing constituent. It enhances the mean grey level of a local area. Speckle noise in SAR is usually a disturbance, inducing troubles for image interpretation. It is made by consistent processing of dispersed signals from multiple disseminated destinations. In SAR oceanography (Kent et al., 2004), for example, speckle noise is induced by signals from elementary spread outs, the gravity-capillary ripples and proved as a base image, underneath the image of the sea waves.

This speckle noise can be expressed by random measure multiplications with pixel measures of the noisy image and can be given as:

$$
P=A+n^{*} A
$$

Where:

$\mathrm{P}=$ The speckle noise distribution image,

$\mathrm{A}=$ The image that is the input and

$\mathrm{n}=$ The uniform noise of image by mean 0 and variance $\mathrm{v}$. 


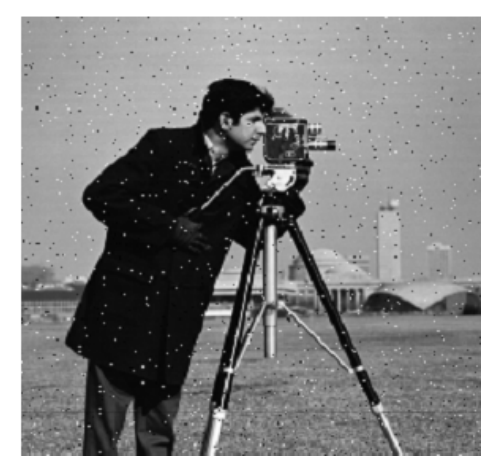

(a)

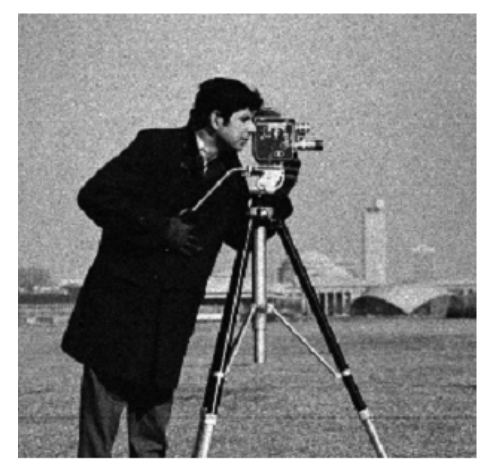

(c)

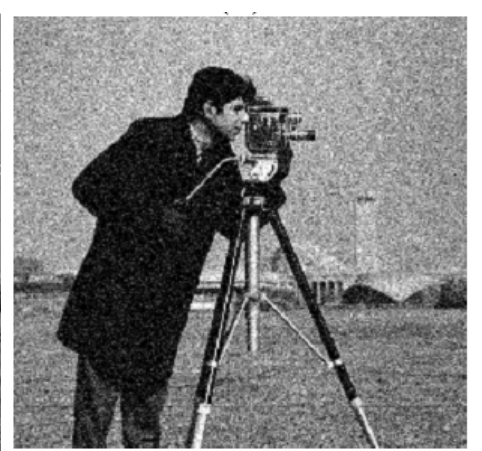

(b)

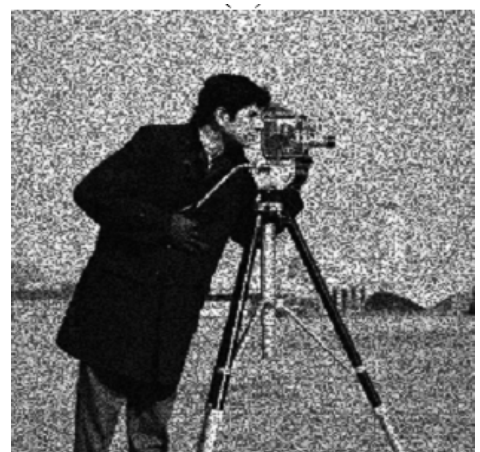

(d)

Fig. 1. Types of noises (a) Salt and pepper noise (b) Gaussian noise (c) Poisson noise (d) Speckle noise

\section{Filters}

Filtering or de-noising in an image processing is a fundamental function which is employed to attain several tasks like interpolation, reduction of noise and re-sampling. De-noising image information is an ideal process employed in almost entire image processing systems. The selection of filter is decided by the nature of the filter task that it is performed by and activity and data type. Filters are needed to eliminate noise from digital images when preserving the details of images kept is an essential part of image processing technique. Here, we preferred two types of filters namely, mean filter and median filter.

\section{Mean Filter}

As we prefer to employ linear filtering to eliminate certain types of image noise and certain filters like Average or Gaussian filters which are reserve for such intension. For instance, an average filter is practicable for eliminating grain noise from a photographic image. Since each pixel has set to the pixel's average in their neighborhood, local fluctuations induced by grain that are eliminated. Schematically linear filtering Algorithms were employed for digital image processing technique. The basic and the most uncomplicated of those algorithms is the Mean Filter which is specified in (Church et al., 2008). The Mean Filter is a type of linear filter that employs a mask on each image pixel in the signal. From each one of the pixel components that come under the mask which are averaged together to build a single pixel. Such Median filter is also known as average filter. The Mean Filter provides poor performance in edge preserving. The Mean filter is defined by:

$$
\text { Mean filter }\left(a_{1} \ldots . . a_{N}\right)=-{ }_{N}^{N} \sum_{i=1} a_{i}
$$

where, $\left(a_{1} \ldots . a_{N}\right)$ is the pixel range of an image. Usually linear filters are employed for noise inhibition.

\section{Median Filter}

The Median filter is a type of nonlinear digital filter which is frequently employed to eliminate image noise. This noise elimination is a distinctive preprocessing measure to enhance the consequences of subsequent processing like edge detection on an image. Median filtering is widely employed in digital image processing techniques since under particular conditions; it maintains edges while eliminating noise. The major thought of the median filter is to operate by the signal entry by entry, substituting each and every entry with their median of neighboring filter entries. Observe that if the window contains odd number entries, so the median is very simple to specify: It is exactly the centre value behind whole entries in the window are classified in a numeric manner. By considering the even number entries, there is more than one median is possible and 
such median filter is also a robust filter. Median filters are broadly employed as smoothers for digital image processing techniques, as well as it is used in digital signal processing and processing of time series. The major benefit of the median filtering technique throughout linear filters is which the median filter can able to remove the impression of input noise values with tremendously large magnitudes (Church et al., 2008; Hwang and Haddad, 1995). The result q of the median filtering at the instant $r$ is estimated as the input values of the median representing to the instants neighboring to $r$ :

$$
\mathrm{q}(\mathrm{r})=\operatorname{median}\left(\left(\mathrm{x}(\mathrm{r}-\mathrm{T} / 2), \mathrm{x}\left(\mathrm{r}-T_{1}+1\right), \ldots, \mathrm{x}(\mathrm{r}+\mathrm{T} / 2)\right)\right.
$$

where, $r$ is the window size of the median filter. Likewise the one-dimensional median filter depicted above and in general two-dimensional filters are employed in digital image processing. Usually images are symbolized in discrete pattern as two dimensional arrays of the image components or pixels:

$$
a=1, \ldots, M_{y}(\text { rows }) \text { and } b=1, \ldots, M_{y}(\text { column })
$$

where, the components $P_{a b}$ are the scalar values and there are several approaches are present for color image processing, where each and every pixel is symbolized by various values, for example, the 'red', 'green', 'blue' values deciding the pixel color.

\section{Performance Metrics}

For equating master image and uncompressed image, we estimate following arguments:

\section{Mean Square Error (MSE)}

The MSE is the Mean Square Error is also known as Cumulative Square Error between the master and the encoded image specified by:

$$
\text { Mean Square Error }=\frac{1}{a b} \sum_{0}^{a=1} \sum_{0}^{b=1}\|p(i, j)-q(i, j)\|^{2}
$$

where, $\mathrm{p}$ is the master image and $\mathrm{q}$ is the uncompressed image. The proportion of the images is $\mathrm{m} \times \mathrm{n}$ and therefore the value of MSE must be as low as possible for efficient compression.

\section{Peak Signal to Noise Ratio (PSNR)}

Peak signal to Noise ratio is the ratio between maximum possible signal power and the power of deforming noise that impresses the quality of their representation. It is determined by:

$$
\text { Peak Signal to Noise Ratio }(P S N R)=20 \log _{10}\left(\frac{\max _{p}}{\sqrt{M S E}}\right)
$$

where, $\max _{p}$ is the signal value at the maximum level that is in our master image.

\section{Conclusion}

Image enhancement of noise affected image is an essential task in digital image processing technique. Filters are employed most effective for elimination of noise from the transmitted images. Here, we depict several noise models and filtering or de-noising techniques. Filtering techniques are splitted into two components linear and non-linear approaches. Later analyzing linear and non-linear filtering each of them comprises benefits and retreats. The determination to implement which special filter is established on the various noise levels at the several test pixel position or operation of the filtering approach on a filtering mask. In future, we will preserve the image edges by implementing Wiener Filter in Wavelet Domain.

\section{Funding Information}

The authors have no support or funding to report.

\section{Author's Contributions}

All authors equally contributed in this work.

\section{Ethics}

This article is original and contains unpublished material. The corresponding author confirms that all of the other authors have read and approved the manuscript and no ethical issues involved.

\section{References}

Boncelet, C., 2005. Image Noise Models. In: Handbook of Image and Video Processing, Bovik, A.C. (Ed.,).

Church, J.C., Y. Chen and S.V. Rice, 2008. A spatial median filter for noise removal in digital images. IEEE Southeastcon. DOI: $10.1109 /$ SECON.2008.4494367

Gonzalez, R.C. and R.E. Woods, 2005. Digital Image Processing. 2nd Edn., Pearson Education.

Hwang, H. and R.A. Haddad, 1995. Adaptive median filters: New algorithms and results. IEEE Trans. Image Proc., 4: 499-502. DOI: 10.1109/83.370679

Jain, A.K., 1989. Fundamentals of Digital Image Processing. 7th Edn., Prentice Hall, Englewood Cliffs, ISBN-10: 0133325784, pp: 569.

Kent, S., O.N. Oçan and T. Ensari, 2004. Speckle reduction of synthetic aperture radar images using wavelet filtering. Proceedings, of the 5th European Conference on Synthetic Aperture Radar, May 2527, Ulm, Germany.

Srinivasan, K.S. and D. Ebenezer, 2007. A new fast and efficient decision-based algorithm for removal of high-density impulse noises. IEEE Signal Proc. Lett., 14: 189-192.

DOI: 10.1109/LSP.2006.884018 